\title{
Clinical Features of 66 Lymphoma Patients Presenting with a Fever of Unknown Origin
}

\author{
Jing Zhang, Bobin Chen, Xiaoping Xu, Zhiguang Lin, Bo Huang, Jing Song and Guowei Lin
}

\begin{abstract}
Objective To investigate the clinical characteristics, diagnostic approaches, short-term efficacy of treatment and prognosis of lymphoma patients presenting with a fever of unknown origin (FUO).

Methods We reviewed the records of 132 patients finally diagnosed with lymphoma in Huashan Hospital, half of whom initially presented with a FUO. The other 66 lymphoma patients without a history of FUO were diagnosed within a month when several patients in the FUO group were also diagnosed.

Results The patients presenting with a FUO were predominantly young men $(71.21 \%, \mathrm{p}=0.35)$ characterized by a temperature $\geq 39^{\circ} \mathrm{C}(55 / 66,83.33 \%)$. Compared with the non-FUO group, patients in the FUO group more often had pancytopenia and hypohepatia, $61.54 \%$ with hypoalbuminemia $(\mathrm{p}<0.0001), 15.50 \%$ with significantly elevated lactate dehydrogenase (LDH) $(\mathrm{p}<0.0001), 92.45 \%$ with elevated serum $\beta_{2}$ microglobulin ( $\mathrm{p}=0.017), 93.48 \%$ with elevated urine $\beta_{2}$ microglobulin $(\mathrm{p}=0.002)$ and $30.77 \%$ with elevated alkaline phosphatase $(\mathrm{p}=0.001)$. Ninety-four percent of the FUO patients had aggressive lymphomas $(\mathrm{p}=0.012)$, with a poor performance status $(96.97 \%, \mathrm{p}=0.003)$, stage III/IV disease $(96.97 \%, \mathrm{p}<0.0001)$, night sweats $(21.21 \%, \mathrm{p}=0.026)$, unexplained weight loss $(46.97 \%, \mathrm{p}=0.002)$ and more than one extranodal site involved (65.15\%, $\mathrm{p}=0.002)$. The patients in the FUO group also showed poor prognoses, and most of them were in the high-intermediate or high risk classification of the disease $(96.61 \%, \mathrm{p}<0.0001)$, with a low complete remission (CR) rate $(61.11 \%$ vs. $93.75 \%, p=0.043)$. Twenty-one $(15.91 \%)$ of all the patients were diagnosed based on the finding of lesion sites by Positron Emission Tomography/Computed Tomography (PET/CT) scanning, which had not been detected by conventional scans.

Conclusion Lymphoma presenting as FUO has a rapid progression and poor prognosis, and is difficult to diagnose. PET/CT scans can provide complementary information for an etiological diagnosis of a FUO and biopsy examinations are significant to establish an early diagnosis for patients presenting with a FUO.
\end{abstract}

Key words: fever of unknown origin, lymphoma, etiology, prognosis

(Intern Med 51: 2529-2536, 2012)

(DOI: 10.2169/internalmedicine.51.7817)

\section{Introduction}

A fever of unknown origin (FUO) was defined by Petersdorf and Beeson in 1961 as a temperature $\geq 38.3^{\circ} \mathrm{C}\left(101^{\circ} \mathrm{F}\right)$ on several occasions, lasting more than 3 weeks, with a diagnosis that remained uncertain after one week of inhospital diagnostic workup (1). In 1991, Durack and Street modified the initial definition: (i) providing distinctions among classical FUO, nosocomial, neutropenic and Human Immunodeficiency Virus (HIV)-associated FUO, and (ii) no established diagnosis after three outpatient visits or three days (instead of one week) of in-hospital investigation (2).

The etiological spectrum of FUO is wide, including infections, non-infectious inflammatory diseases (NIID), malignancies and miscellaneous conditions (3-5). Non-Hodgkin's lymphoma (NHL) is the main cause of FUO among malignancies, and is associated with greater difficulty in establishing a definite diagnosis of patients with fever compared with infections and NIID $(6,7)$. This is partially due to the fact that the clinical features of lymphoma presenting as a FUO have not yet been clearly elucidated. 
Table 1. Clinical Features of the FUO Group

\begin{tabular}{ll}
\hline Characteristics of Fever & No. $(\%)$ \\
\hline Temperature & \\
$\quad$ Moderate degree fever & $11(16.67)$ \\
$\quad$ High Fever & $55(83.33)$ \\
Duration & \\
$\quad \leq 3$ months & $44(66.67)$ \\
$3-6$ months & $17(25.76)$ \\
$\quad>6$ months & $5(7.58)$ \\
Fever type & \\
Continuous fever & $1(1.52)$ \\
Undulant fever & $3(4.55)$ \\
Remittent fever & $4(6.06)$ \\
Intermittent fever & $4(6.06)$ \\
Irregular fever & $54(81.82)$ \\
With rigors & $11(16.67)$ \\
\hline
\end{tabular}

Table 2. Comparison of the Clinical Features between the Groups

\begin{tabular}{lcccc}
\hline Clinical Features & $\begin{array}{c}\text { FUO } \\
\text { No. }(\%)\end{array}$ & $\begin{array}{c}\text { Non-FUO } \\
\text { No. }(\%)\end{array}$ & $\chi^{2}$ & p \\
\hline Performance status $>1$ & $64(96.97)$ & $53(80.3)$ & 9.10 & 0.003 \\
Stage III/IV & $64(96.97)$ & $49(74.24)$ & 13.83 & 0.000 \\
Night sweats & $14(21.21)$ & $5(7.58)$ & 4.98 & 0.026 \\
Weight loss in the previous 6 & $31(46.97)$ & $14(21.21)$ & 9.74 & 0.002 \\
months $>10 \%$ & & & & \\
Multiple dropsy of serous cavity & $5(7.58)$ & $4(6.06)$ & - & 1.000 \\
Hemophagocytic syndrome & $10(15.15)$ & $0(0)$ & 10.82 & 0.001 \\
More than one extranodal site & $43(65.15)$ & $25(37.88)$ & 9.83 & 0.002 \\
\hline
\end{tabular}

We collected the clinical data of patients presenting with a FUO who were hospitalized in our hospital during the last 12 years who were finally diagnosed with lymphoma, and for comparison, the data from lymphoma patients without a history of FUO who were diagnosed in a month when patients in the FUO group were also diagnosed.

In the present study, we retrospectively explored the clinicopathological characteristics of lymphoma presenting as a FUO, and compared the differences between these patients and conventional lymphoma patients in terms of the clinical features, diagnostic approaches used, and short-term efficacy of treatment. The present report will be useful for physicians coping with the difficulty in diagnosing lymphoma presenting as a FUO in clinical practice.

\section{Materials and Methods}

\section{Patient selection}

We reviewed the records of 132 patients finally diagnosed with lymphoma according to a pathological diagnosis between April 1998 and January 2011 at our hospital, half of whom fulfilled the criteria for a FUO. The remaining 66 lymphoma patients without a history of FUO were diagnosed during a month when patients in the FUO group were also diagnosed. The pathological diagnosis was made and reviewed according to the World Health Organization (WHO) classification of tumors of hematopoietic and lym- phoid tissues (8).

\section{Data collection}

Detailed information about the patients included in the study was collected, including their clinical characteristics (gender, age, fever features, concomitant symptoms, sites of lesions and interventions before diagnosis), results of laboratory tests (complete blood count, liver function, etc.), imaging studies such as B-ultrasonic, CT, magnetic resonance imaging (MRI), or Positron Emission Tomography/Computed Tomography (PET/CT) scans, pathological evaluation methods and short-term efficacy of treatment.

\section{Statistical analyses}

The differences between the two groups were analyzed by the chi-square test or Fisher's exact test as appropriate for categorical data, unpaired Student's $t$-test for continuous variables, and with Fisher exact tests for categorical variables. A value of $\mathrm{p}<0.05$ was consider to indicate statistical significance. All statistical analyses were carried out with the STATA software program (version 10).

\section{Results}

\section{Clinical features}

\section{Gender and age}

We collected the case records of 66 patients initially presenting with a FUO and 66 patients without a history of fever, consisting of 89 men and 43 women. There was no significant difference in the gender composition between the FUO group and the non-FUO group $\left(\chi^{2}=0.86, \mathrm{p}=0.35\right)$. The patients in the FUO group were younger at diagnosis (44.39 \pm 16.8 vs. $54.8 \pm 15.98$ years old, $\mathrm{t}=-3.64, \mathrm{p}=0.0004)$ than those in the non-FUO group, and fewer cases in the former group were older than 60 years $\left(\chi^{2}=9.78, \mathrm{p}=0.002\right)$.

\section{Clinical features of patients presenting with a FUO}

Most patients in the FUO group had a body temperature $\geq$ $39^{\circ} \mathrm{C}$ without signs of regional infection. The type of fever was predominately an irregular fever. The median duration of fever before diagnosis was two months (range 0.7-60 months). Some patients had concomitant chills (Table 1).

\section{General condition}

There were significant differences in the clinical features between patients in the FUO group and the non-FUO group (Table 2). The Eastern Collaborative Oncology Group (ECOG) score was used to measure the performance status (PS) of patients (9). The staging classification was determined by Ann-Arbor staging. The patients in the FUO group had more aggressive clinical features: a poor performance status, advanced stage, night sweats, unexplained weight loss and hemophagocytic syndrome (HPS), despite 


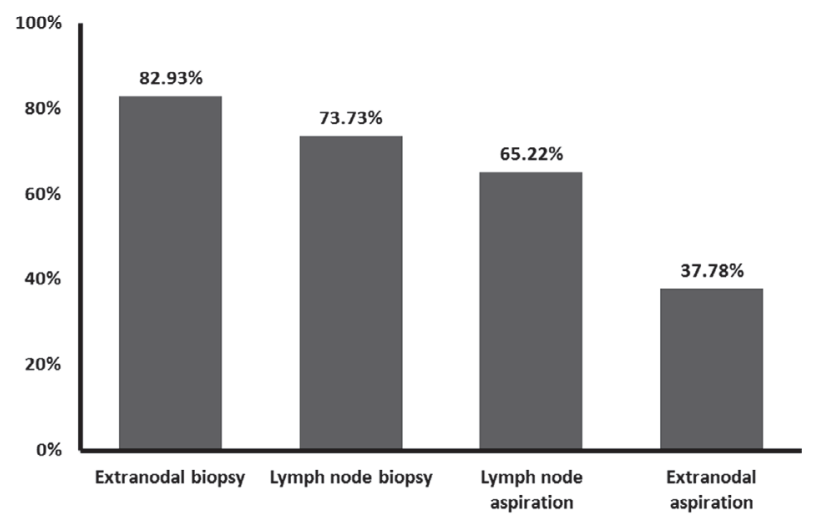

Figure 1. Comparison of the positive rates of detection by different diagnostic approaches.

Table 3. Interventions before Diagnosis

\begin{tabular}{cc}
\hline Interventions & FUO \\
& No. (\%) \\
\hline Antibiotic therapy & $54(81.82)$ \\
$\begin{array}{l}\text { A-lactams } \\
\text { Aminoglycosides }\end{array}$ & $49(74.24)$ \\
Macrolides & $7(10.61)$ \\
Lincomycin & $15(22.73)$ \\
Hexapeptide & $7(10.61)$ \\
Tetracyclines & $11(16.67)$ \\
Quinolones & $4(6.06)$ \\
Nitromidazoles & $33(0.50)$ \\
Fosfomycin & $8(12.12)$ \\
Furan & $6(9.09)$ \\
Curative effect* & $2(3.03)$ \\
Temperature drops & $0(0)$ \\
Repeated fever & $25(46.30)$ \\
No effects & $28(51.85)$ \\
Adrenocortical hormone therapy & $31(46.97)$ \\
Curative effect & \\
Temperature drops & $0(0)$ \\
Repeated fever & $24(77.42)$ \\
No effects & $7(22.58)$ \\
Anti-tuberculous therapy & $7(10.61)$ \\
\hline 5 & patients (81.82\%) among the FUO group received \\
withdrew antibiotic drugs because of drug allergy and the \\
statistic was not counted in the curative effect.
\end{tabular}

their lower age distribution.

\section{Distribution of lesion locations}

There were no significant differences in the lesion distribution between the FUO group and the non-FUO group. Nodal and extranodal involvement were prevalent in both groups $\left(\chi^{2}=3.27, \mathrm{p}=0.07\right)$ and most patients had superficial lymph nodes enlargement $\left(\chi^{2}=0.57, \mathrm{p}=0.45\right)$ and multi-site involvement $\left(\chi^{2}=0.47, \mathrm{p}=0.49\right)$. However, compared with the non-FUO group, the frequency of patients with more than one extranodal site was found to be higher in the FUO group (Table 2).

\section{Lymph node involvement}

There was no significant difference between the two groups in the overall distribution of involved lymph nodes

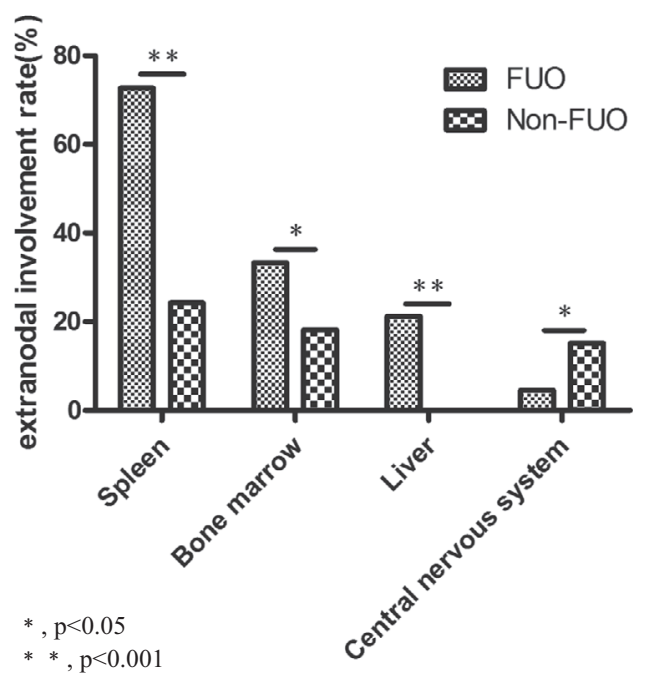

Figure 2. Comparison of extranodal involvement between the groups.

$\left(\chi^{2}=4.20, \mathrm{p}=0.65\right)$, with both groups having extensive involvement. Forty-six $(69.7 \%)$ of the patients with a history of FUO had axillary lymph node enlargement and 41 $(62.12 \%)$ had inguinal lymph node involvement. The mediastinal and retroauricular lymph nodes were less frequently involved.

\section{Extranodal involvement}

The spleen, bone marrow and liver were common sites of extranodal involvement in the FUO group, and central nervous system involvement was uncommon (Fig. 2). Other rare sites included the nasopharynx, gastrointestinal tract, skin/ subcutaneous tissue, adrenal glands, bone, oropharynx, mediastinum, tonsils, thyroid, breasts, shoulder joints, lungs, chest wall, ascites, salivary glands, orbits, testes, parotid glands, kidneys, pericardium, and tongue.

\section{Interventions before diagnosis}

Lymphoma presenting as a FUO remains a challenge in clinical practice. In the present study, fifty-one patients (77.27\%) in the FUO group were transferred to our hospital for a definite diagnosis and further treatment. Fifty-four $(81.82 \%)$ of the patients in the FUO group had received empiric antibiotic therapy before the diagnosis, which had included mostly $\beta$-lactam and quinolone agents. One of the patients had stopped treatment with the antibiotic drugs because of a drug allergy. Thirty-one patients $(46.97 \%)$ received adrenocortical hormone and seven patients $(10.61 \%)$ had received four anti-tuberculosis medications (ethambutol, rifampin, isoniazid, and pyrazinamide) before their diagnoses of lymphoma. Because of the inconspicuous lesion sites and/or difficulty in obtaining a biopsy at the onset of the disease, antibiotic therapy, hormone therapy and antituberculosis treatment, resulting in poor efficacy, were prevalent in the lymphoma patients presenting with a FUO before the definite diagnosis. Lymphoma was ultimately confirmed by the presence of palpable lesions and a local 
Table 4. Comparison of the Results of Laboratory Evaluations

\begin{tabular}{lcccc}
\hline Laboratory evaluations & $\begin{array}{c}\text { FUO } \\
\text { No. }(\%)\end{array}$ & $\begin{array}{c}\text { Non-FUO } \\
\text { No. }(\%)\end{array}$ & $\chi^{2}$ & $\mathrm{p}$ \\
\hline Leucocytopenia & $27(40.91)$ & $13(20)$ & 6.75 & 0.009 \\
Neutropenia & $16(25)$ & $3(4.69)$ & 10.45 & 0.001 \\
Lymphopenia & $41(63.08)$ & $20(31.25)$ & 13.10 & 0.000 \\
Low hemoglobin & $50(76.92)$ & $13(20)$ & 42.16 & 0.000 \\
Thrombocytopenia & $20(30.30)$ & $9(13.85)$ & 5.15 & 0.023 \\
Hepatic dysfunction & & & & \\
$\quad$ Hypoproteinemia & $40(61.54)$ & $7(10.77)$ & 36.29 & 0.000 \\
$\quad$ Elevated alanine transaminase & $24(36.36)$ & $6(9.09)$ & 13.98 & 0.000 \\
$\quad$ Elevated aspartate transaminase & $34(52.31)$ & $13(20.00)$ & 14.70 & 0.000 \\
$\quad$ Elevated total bilirubin & $6(9.09)$ & $7(10.61)$ & 0.085 & 0.77 \\
$\quad$ Elevated direct Bilirubin & $10(15.38)$ & $4(6.25)$ & 2.78 & 0.095 \\
Lactate dehydrogenase $>1.5$ fold & $25(43.10)$ & $5(9.62)$ & 15.50 & 0.000 \\
Elevated serum $\beta_{2}$ microglobulin & $49(92.45)$ & $40(75.47)$ & 5.67 & 0.017 \\
Elevated urine $\beta_{2}$ microglobulin & $43(93.48)$ & $75(68.09)$ & 9.60 & 0.002 \\
Elevated alkaline phosphatase & $20(30.77)$ & $5(7.69)$ & 11.14 & 0.001 \\
Elevated immunoglobulin & & & & \\
$\quad$ IgG & $8(18.18)$ & $4(33.33)$ & - & 0.26 \\
$\quad$ IgA & $9(20)$ & $0(0)$ & - & 0.18 \\
$\quad$ IgM & $6(13.33)$ & $2(16.67)$ & - & 0.67 \\
Erythrocyte sedimentation rate & $29(67.44)$ & $4(40)$ & - & 0.15 \\
\hline
\end{tabular}

biopsy for pathological examination.

\section{Laboratory evaluations}

Compared with the patients in the non-FUO group, those in the FUO group more frequently exhibited cytopenia in one or more hematopoietic cell lineages and hepatic dysfunction, with an elevated serum lactate dehydrogenase level over 1.5 -fold the upper limit, increased $\beta_{2}$ microglobulin and alkaline phosphatase (Table 4). Forty-four patients (66.67\%) in the FUO group had culture-negative blood test results obtained repeatedly before the definite diagnosis.

\section{Imaging studies}

Twenty-one $(15.91 \%)$ of all of the patients were found to have lesion sites by PET/CT scans, which had not been detected by conventional scanning methods such as type-B ultrasonic, CT and MRI scans, including 13 patients in the FUO group and 8 patients in the non-FUO group. The most common newfound lesion sites were the mediastium (8 patients), lymph nodes in deep locations (5 patients), bones (3 patients), bone marrow ( 3 patients), lung and hilus pulmonis (3 patients), superficial lymph nodes (2 patients), adrenal glands ( 2 patients), spleen (2 patients), thyroid (2 patients), and nasopharynx (2 patients). Lesions located in the tonsils, shoulder joints, hypophysis, hip joints, skin/subcutaneous tissue, kidneys, adrenal glands and pericardium were rarer. The median long axis of the enlarged spleen of patients with a FUO and without a FUO was $130 \mathrm{~mm}$ (range 102-222 $\mathrm{mm}$ ) and $116.5 \mathrm{~mm}$ (range 102-190 mm), respectively (U= $1.15, \mathrm{p}=0.25)$.

No significant difference was found in the median maximum standard uptake value (SUV) between the FUO group and the non-FUO group, with values of 14.9 and 23.75 , respectively ( $\mathrm{U}=-0.94, \mathrm{p}=0.35$ ). The mean $\mathrm{SUV}_{\max }$ of aggressive B-cell lymphomas $(22.72 \pm 13.26)$ was higher than that of T/Natural killer $(\mathrm{NK})$-cell lymphomas $(13.10 \pm 8.38)$ in the FUO group ( $\mathrm{t}=1.78, \mathrm{p}=0.094)$. The median $\mathrm{SUV}_{\max }$ of $\mathrm{B}-$ cell lymphomas in the FUO group and the non-FUO group were 25.60 and 25.50 , respectively ( $\mathrm{U}=-0.24, \mathrm{p}=0.81$ ). The median $\mathrm{SUV}_{\max }$ values of aggressive lymphomas had a tendency to be higher than those of indolent lymphomas, with values of 16.35 and 3.90 , respectively $(U=1.79, p=0.07)$.

\section{Diagnostic approaches and pathological findings}

Among the methods used for puncture and biopsy examinations in all patients, extranodal biopsy had the highest positive rate for a diagnosis (Fig. 1). The median time between patients noticing lymph node adenopathy and seeking medical attention with a lymph node biopsy was 1.5 months (range 0.23-60 months).

\section{Biopsy site selection}

Cervical lymph nodes $\left(\chi^{2}=0.81, \mathrm{p}=0.37\right)$ were the most frequently targeted site for lymph node biopsy in the FUO group, followed by inguinal, submaxillary, retroperitoneal, axillary, supratrochlear, and opisthotic lymph nodes, and one unknown site. Forty-eight $(72.73 \%)$ of the patients in the FUO group underwent bone marrow aspiration, which was significantly higher than the number of patients in the nonFUO group $\left(\chi^{2}=4.00, p=0.046\right)$, while pelvic, adrenal gland and liver aspiration were rarely performed in either group. Lymphoma can involve essentially any organ in the human body. However, the bone marrow $\left(\chi^{2}=1.69, \mathrm{p}=0.19\right)$ and skin $\left(\chi^{2}=0.085, \mathrm{p}=0.77\right)$ were the preferred extranodal sites to biopsy. Other locations included the nasopharynx, central nervous system, chest wall, pharynx, breasts, salivary glands, tonsils, orbits, thyroid, testes, parotid glands, skin/ subcutaneous tissue, shoulder joints and tongue. Out of the total 132 patients, 28 patients $(21.21 \%)$ underwent an abdominal laparotomy, which thus led to a definite diagnosis. The most common sites of involvement identified by the laparotomy were the ileocolon $\left(\chi^{2}=0.12, p=0.73\right)$, retroperito- 
Table 5. Comparison of the Pathological Results between the Groups

\begin{tabular}{|c|c|c|c|c|}
\hline Pathologies & $\begin{array}{c}\text { FUO } \\
\text { No. }(\%)\end{array}$ & $\begin{array}{l}\text { Non-FUO } \\
\text { No. }(\%)\end{array}$ & $\chi^{2}$ & $\mathrm{p}$ \\
\hline B-cell & $25(37.88)$ & $58(87.88)$ & 35.30 & 0.000 \\
\hline Diffuse large B-cell lymphoma & $22(33.33)$ & $33(50)$ & 3.77 & 0.052 \\
\hline Follicular lymphoma & $0(0.00)$ & $4(6.06)$ & - & 0.12 \\
\hline $\begin{array}{l}\text { Extranodal marginal zone } \\
\text { lymphoma of mucosa-associated } \\
\text { zone lymphoma }\end{array}$ & $0(0.00)$ & $4(0.00)$ & - & 0.12 \\
\hline \begin{tabular}{ll}
\multicolumn{1}{c}{ Chronic } & lymphocytic \\
leukaemia/small & lymphocytic \\
lymphoma &
\end{tabular} & $0(0.00)$ & $3(4.55)$ & - & 0.24 \\
\hline Unclassification & $3(4.55)$ & $10(15.15)$ & 4.18 & 0.04 \\
\hline T/NK-cell & $29(43.94)$ & $6(9.09)$ & 20.57 & 0.000 \\
\hline $\begin{array}{l}\text { Anaplastic large cell } \\
\text { lymphoma }\end{array}$ & $7(10.60)$ & $2(3.03)$ & - & 0.16 \\
\hline $\begin{array}{l}\text { Peripheral T-cell lymphoma, } \\
\text { NOS }\end{array}$ & $6(9.09)$ & $2(3.03)$ & - & 0.27 \\
\hline $\begin{array}{c}\text { Extranodal } \\
\text { lymphoma, nasal type }\end{array}$ & $4(6.06)$ & $0(0.00)$ & - & 0.12 \\
\hline $\begin{array}{l}\text { Enteropathy-associated T-cell } \\
\text { lymphoma }\end{array}$ & $4(6.06)$ & $0(0.00)$ & - & 0.12 \\
\hline Unclassification & $3(4.55)$ & $1(1.52)$ & - & 0.62 \\
\hline Unclassification & $11(16.67)$ & $1(1.52)$ & 6.91 & 0.009 \\
\hline Aggressive lymphoma & $45(93.75)$ & $41(75.93)$ & 6.10 & 0.013 \\
\hline
\end{tabular}

Table 6. Comparison of the International Prognostic Index Scores

\begin{tabular}{lcccc}
\hline IPI* & FUO & Non-FUO & $\chi^{2}$ & $p$ \\
& No. (\%) & No. $(\%)$ & & \\
\hline$<2$ & $0(0.00)$ & $26(48.15)$ & - & 0.000 \\
2 & $21(35.59)$ & $16(29.63)$ & 0.46 & 0.50 \\
3 & $28(47.46)$ & $6(11.11)$ & 17.71 & 0.000 \\
4 & $6(10.17)$ & $6(11.11)$ & 0.026 & 0.87 \\
5 & $4(6.78)$ & $0(0)$ & - & 0.12 \\
Low \&Low intermediate & $2(3.40)$ & $34(53.70)$ & 46.09 & 0.000 \\
High intermediate \&High & $57(96.61)$ & $20(37.04)$ & 46.09 & 0.000 \\
\hline * IPI: international prognostic index & & &
\end{tabular}

neal lymph nodes $\left(\chi^{2}=1.36, \mathrm{p}=0.24\right)$, stomach $\left(\chi^{2}=3.07, \mathrm{p}=\right.$ $0.080)$ and liver $\left(\chi^{2}=4.13, \mathrm{p}=0.042\right)$, while the kidneys, adrenal glands, ascites, abdominal wall and spleen were rarely affected.

\section{Pathological findings}

There were 65 cases of NHL and 1 case of Hodgkin's lymphoma in the FUO group. Most of these were categorized as aggressive lymphomas, mainly T/NK-cell lymphomas, including rare pathological categories of subcutaneous panniculitic T-cell lymphoma, angioimmunoblastic T-cell lymphoma and primary cutaneous aggressive epidermotropic cytotoxic T-cell lymphoma. Diffuse large B-cell lymphoma (DLBCL) was predominant in the B-cell lymphomas of the FUO group (Table 5). DLBCL was also the most common type in the non-FUO group, with there being rarer cases of mantle cell lymphoma, precursor lymphocytic lymphoma and nodal marginal zone lymphoma.

\section{Prognosis}

Of the 52 patients in the FUO group who received che- motherapy, 37 patients $(71.15 \%)$ had abatement of the fever after chemotherapy, which occurred after one day in 22 patients, two days in eight patients, three days in four patients, and after four days in three patients. The international prognostic index (IPI) score for patients in the FUO group was significantly higher than that for patients in the non-FUO group (Table 6). Of the patients who received long-term treatment in our hospital, 11 patients in the FUO group reached complete remission $(\mathrm{CR})$ in one year; five died shortly after their diagnoses; one patient relapsed after two rounds of autologous stem cell transplantation, and one patient went into a coma and was no longer administered therapy. Fifteen patients in the non-FUO group reached a CR in one year, with 1 case of death occurring shortly after diagnosis.

\section{Discussion}

Fever is a common symptom of lymphoma, and the possible mechanisms underlying this feature may include: (i) an increase of cytokines, such as tumor necrosis factor (TNF)$\alpha$, that regulate the fever reaction through the hypothalamus (10); (ii) indirect factors, such as opportunistic infections caused by the immunodeficiency that develops during the course of the disease. We found that patients presenting with a FUO were predominantly young adults; fewer patients were older than 60 years than in the non-FUO group. There was a tendency for the patients to be younger in our cohort compared with previous reports $(11,12)$. The median age of patients diagnosed with T/NK-cell NHL and B-cell NHL in Shanghai was reported to be 49 and 58 years, respectively (13). The prevalence of T/NK-cell lymphomas in the FUO group may have contributed to the tendency for 
our patients to be younger. The gender of our patients was consistent with those described in previous studies of lymphoma, with a male predominance.

Patients presenting with a FUO often had a high fever at onset and a long duration of fever before diagnosis. The type of fever was frequently irregular, with chills and undefined lesions, which led to difficulty in making a diagnosis and consequently delayed the treatment. Some of the patients had concomitant HPS in our study. HPS is usually associated with NHL, and is referred to as lymphomaassociated hemophagocytic syndrome (LAHS).

It remains a formidable challenge to establish the histological diagnosis of lymphoma presenting as FUO because of the inconspicuous or deep lymphadenopathy at the onset of the disease, despite the recent advances in diagnostic techniques. Imaging provides key information about precise staging to define the local lesion and distant organ involvement. However, conventional techniques only deliver localized information, and the use of total body CT and MRI are not widespread. Previous studies revealed that PET contributed to the etiological diagnosis in $16 \%-69 \%$ of all patients with FUO (14-21), with a positive predictive value of $80 \%$, negative predictive value of $50 \%$, sensitivity of $89 \%$, and specificity of $33 \%$ (22).

In addition, PET/CT scanning plays a complementary role in the etiological diagnosis of FUO (23). In the absence of palpable lymphadenopathy, PET/CT is valuable for the metabolic assessment of a normal-sized lymph node, which would otherwise be dismissed as normal on conventional imaging $(24,25)$. In the present study, 21 patients $(15.91 \%)$ were found to have lesions by PCT/CT that had been overlooked by conventional imaging examinations such as Bultrasonic, CT and MRI scans. This was especially true for lesions in the mediastium or deep lymph nodes. These results suggest that PET/CT provides a more comprehensive and effective value in disease assessment and staging compared with other imaging examinations, especially when the results of conventional imaging are normal or unable to distinguish benign from malignant lesions $(26,27)$.

Extranodal tissue biopsy and lymph node biopsy had higher positive rates than other puncture and biopsy examinations. We found that there was a long time span between when patients noticed lymph node adenopathy and sough medical help, indicating that most patients in our study did not undergo timely biopsy examinations for a definite diagnosis. Therefore, the performance of a lymph node biopsy should be promoted to establish an accurate diagnosis due to its high positive rate and minimal tissue injury, once suspicious lesions are found by imaging studies. Alam (28) and colleagues reported that the diagnostic sensitivity of aspiration cytology for NHL was $95.4 \%$. However, this was not in accordance with our clinical practice. In our study, only 15 patients $(68.18 \%)$ had a diagnosis clarified by lymph node aspiration, which was not as sensitive as lymph node biopsy $\left(\chi^{2}=20.06, p<0.0001\right)$. The difference may be due to the application of immunohistochemistry, flow cytometry, and mo- lecular studies, such as polymerase chain reaction and fluorescent in situ hybridization, which increased the diagnostic accuracy. Splenomegaly was common in patients with malignant lymphoma, and splenectomy was one of the important measures used to make a final diagnosis. When patients presenting with a FUO have splenomegaly at presentation in the absence of clinically palpable lymphadenopathy, a splenectomy should thus be considered as a pathological examination.

In the study of Wang and colleagues, lymphomas presenting as FUO were primarily B-cell lymphomas. But in our study, the FUO group mainly comprised T/NK-cell lymphomas, in line with the result of Mao's research (9). In a large scale prospective study in Shanghai, T/NK-cell NHL composed $20.7 \%$ of all NHL cases, which was similar to the $26.72 \%$ in the present study. However, most of the T/NKcell lymphomas were detected in the patients with a FUO. adult T-cell leukaemia/lymphoma (ATLL) cells can produce IL-6 and IFN- $\gamma$, which can cause a fever and activate normal macrophages to secrete TNF- $\alpha$, which can then exacerbate the fever (29). This phenomenon of secretion of cytokines may be especially prevalent in T/NK-cell lymphomas, which would explain why the FUO group included mainly T/NKcell lymphomas. Li reported an analysis of nine cases of splenic T-cell and NK-cell lymphomas, of which eight had a fever (30). Although the spleen is rarely involved in T/NKcell lymphomas, most of the T/NK-cell lymphomas with splenic involvement are detected in the presence of fever. Therefore, we believe that the histological distribution of the lesions in the FUO group not only contributed to the tendency for the patients to be younger than those in previous studies, but also to the high proportion of splenic involvement.

A total of $71.15 \%$ of the patients presenting with a FUO were sensitive to chemotherapy, and one-third of them experienced abatement of the fever the first day after chemotherapy. Given the long course of the disease, difficulty in diagnosis, and poor condition at the time of diagnosis, $81.82 \%$ of the patients presenting with a FUO had been treated with antibiotics before the diagnosis, which included mostly $\beta$ lactam and quinolone agents, $46.97 \%$ had received hormone therapy, and $10.61 \%$ had received anti-tuberculosis therapy. All medical therapy before the diagnosis resulted in no or limited effects. Our research suggests that antibiotic abuse is widespread in clinical practice, and empiric therapy is not appropriate for patients with a FUO, because it might obscure the diagnosis and cause other complications, such as toxicity and antibiotic resistance.

The lymphoma patients with HPS had poor prognoses. In a study of 52 adult patients, $81 \%$ of the patients with LAHS were in poor condition and mainly died of multiple organ failure or disseminated intravascular coagulation (DIC), with a median survival time of only 83 days (31). HPS is common in patients with T/NK-cell lymphomas, such as the nasal type extranodal NK/T-cell lymphoma and primary cutaneous gamma-delta T-cell lymphoma. Patients with T/NK- 
cell lymphomas with HPS have a poorer prognosis than those with aggressive B-cell lymphomas (32-34). DLBCL and intravascular large B-cell lymphoma are common aggressive B-cell lymphomas associated with HPS (35-37). In our study, $15.15 \%$ of the patients presenting with a FUO also had HPS, including two cases of DLBCL, two of anaplastic large cell lymphoma, one of primary cutaneous aggressive epidermotropic cytotoxic T-cell lymphoma, one of angioimmunoblastic T-cell lymphoma and four cases that were unclassified. Twenty percent of these patients died, and none achieved a CR after chemotherapy. In order to make a diagnosis without delay and give appropriate treatment to patients presenting with a FUO, prompt performance of imaging studies, and puncture and biopsy examinations, should be encouraged.

Compared with lymphoma patients in the non-FUO group, the patients presenting with a FUO frequently showed a larger tumor burden, more rapid progression and poorer prognosis as assessed by the IPI score (38). A total of $94 \%$ of the patients in the FUO group had aggressive lymphomas, and showed more aggressive clinical features, including a poor PS, advanced stage, systemic symptoms (night sweats, unexplained weight loss), more than one extranodal lesion site, and HPS. The presence of symptoms (fever, night sweats and weight loss) is a negative prognostic factor in patients with Hodgkin's lymphoma. The relevance of these B symptoms in patients with non-Hodgkin's lymphoma is less clear, but the symptoms tend to be related to more widespread or higher histological grade disease. Several previous large scale studies showed that hypoalbuminemia, elevation of serum $\beta 2$ microglobulin $(39,40)$ and an elevated LDH level were important prognostic factors for lymphoma with a FUO $(41,42)$. Increased alkaline phosphatase activity can be an indirect indicator of progression and a poor prognosis of lymphoma (43). In our study, the laboratory evaluations of patients presenting with a FUO showed significant abnormalities in the levels of LDH, $\beta_{2}$ microglobulin and alkaline phosphatase, often associated with hypoalbuminemia. The FUO group showed a lower CR rate than the non-FUO group. Out of the six patients presenting with a FUO who died of the disease, five had progressive pancytopenia and four had hypohepatia, consistent with the conclusion of a previous study that progressive pancytopenia and hypohepatia indicate a poor prognosis of lymphoma (11). It is regrettable that, due to the retrospective nature of the study, we cannot provide information about the overall survival (OS) and progression free survival (PFS) of the patients to reveal the prognosis of lymphoma patients presenting with a FUO due to the loss of patients to follow-up. Long-term observations of survival are still needed.

Chihara (44) and colleagues revealed that a high $\mathrm{SUV}_{\max }$ value was a significant poor prognostic factor for both PFS and OS in DLBCL, independent of the IPI. We found that the $\mathrm{SUV}_{\max }$ value of T/NK-cell lymphomas was lower than that of aggressive B-cell lymphomas, despite the higher metabolic activity. Another study demonstrated that NK-cell lymphomas have a great deal of coagulative necrosis and inflammatory components, and that they show a relatively slower tumor growth rate compared with aggressive B-cell lymphomas (45). A $\mathrm{SUV}_{\max }>10$ is considered to be a poor prognostic factor for tumors with an aggressive histology (46). However, in our study, the $\mathrm{SUV}_{\max }$ did not reflect an aggressive histology, which might be related to the different pathological composition of the different patient cohorts. The correlation between the $\mathrm{SUV}_{\max }$ and aggressive histology needs to be confirmed in additional large scale studies.

In conclusion, a rapid progression, a difficult diagnosis, and a poor prognosis are all significant clinical features of lymphomas presenting as a FUO, compared with lymphomas without a FUO. PET/CT scanning is a viable tool for patients presenting with a FUO with repeated negative blood culture and no clear infected lesions. The present study should therefore remind physicians that a timely biopsy examination should be considered to clarify the diagnosis and lay a foundation for appropriate treatment in such cases.

\section{The authors state that they have no Conflict of Interest (COI).}

\section{Acknowledgement}

The study was supported by Grant No. 2008064 from the Scientific Fund of Shanghai Health Bureau and Grant No. 10ZR 1405200 from Shanghai Natural Science Fund.

\section{References}

1. Petersdorf RG, Beeson PB. Fever of unexplained origin: report on 100 cases. Medicine 40: 1-30, 1961.

2. Durack DT, Street AC. Fever of unknown origin-reexamined and redefined. Curr Clin Top Infect Dis 11: 35-51, 1991.

3. Colpan A, Onguru P, Erbay A, et al. Fever of unknown origin: analysis of 71 consecutive cases. Am J Med Sci 334: 92-96, 2007.

4. Kucukardali Y, Oncul O, Cavuslu S, et al. The spectrum of diseases causing fever of unknown origin in Turkey: a multicenter study. Int J Infect Dis 12: 71-79, 2008.

5. Cunha BA. Fever of unknown origin: focused diagnostic approach based on clinical clues from the history, physical examination, and laboratory tests. Infect Dis Clin North Am 21: 1137-1187, 2007.

6. Vanderschueren S, Knockaert D, Adriaenssens T, et al. From prolonged febrile illness to fever of unknown origin-the challenge continues. Arch Intern Med 163: 1033-1041, 2003.

7. Wei W, Hesheng L. Malignancy in Chinese adults presenting as fever of unknown origin. Int J Clin Pract 57: 508-512, 2003.

8. Sterdlow SH, Campo E, Harris NL, et al. WHO classification of tumours of haematopoietic and lymphoid tissue. In: Volume 2: IARC WHO classification of tumours, No 2. IARC Press, Lyon, France, 2008.

9. Oken MM, Creech RH, Tormey DC, et al. Toxicity and response criteria of the Eastern Cooperative Oncology Group. Am J Clin Oncol 5: 649-655, 1982.

10. Kurzrock R. The role of cytokines in cancer-related fatigue. Cancer 92 (6 Suppl): 1684-1688, 2001.

11. Mao JF, Liu ZY, Zhao YQ. The diagnosis of non-Hodgkin's lymphoma (NHL) with fever. Beijing Medical Journal 25: 372-374, 2003.

12. Wang $\mathrm{Z}$, Feng JP, Wang Ch, et al. Malignant lymphoma first presenting as fever of unknown origin: a clinical and pathological analysis of 48 cases. Chinese General Pracitce 12: 686-687, 2009. 
13. Gross SA, Zhu X, Bao L, et al. A prospective study of 728 cases of non-Hodgkin lymphoma from a single laboratory in Shanghai, China. Int J Hematol 88: 165-173, 2008.

14. Bleeker-Rovers CP, de Kleijn EM, Corstens FH, et al. Clinical value of FDG PET in patients with fever of unknown origin and patients suspected of focal infection or inflammation. Eur J Nucl Med Mol Imaging 31: 29-37, 2004.

15. Bleeker-Rovers CP, Vos FJ, Mudde AH, et al. A prospective multicentre study of the value of FDG-PET as part of a structured diagnostic protocol in patients with fever of unknown origin. Eur $\mathrm{J}$ Nucl Med Mol Imaging 34: 694-703, 2007.

16. Lockmans D, Knockaert D, Maes A, et al. Clinical value of [(18)F]fluoro-deoxyglucose positron emission tomography for patients with fever of unknown origin. Clin Infect Dis 32: 191-196, 2001

17. Buysschaert I, Vanderschueren S, Blockmans D, et al. Contribution of (18)fluoro-deoxyglucose positron emission tomography to the work-up of patients with fever of unknown origin. Eur J Intern Med 15: 151-156, 2004.

18. Kjaer A, Lebech AM, Eigtved A, et al. Fever of unknown origin: prospective comparison of diagnostic value of 18F-FDG PET and 111In-granulocyte scintigraphy. Eur J Nucl Med Mol Imaging 31: 622-626, 2004.

19. Lorenzen J, Buchert R, Bohuslavizki KH. Value of FDG PET in patients with fever of unknown origin. Nucl Med Commun 22: 779-783, 2001

20. Meller J, Altenvoerde G, Munzel U, et al. Fever of unknown origin: prospective comparison of [18F]FDG imaging with a doublehead coincidence camera and gallium-67 citrate SPET. Eur J Nucl Med 27: 1617-1625, 2000.

21. Bleeker-Rovers CP, Vos FJ, de Kleijn EM, et al. A prospective multicenter study on fever of unknown origin: the yield of a structured diagnostic protocol. Medicine (Baltimore) 86: 26-38, 2007.

22. Sheng JF, Sheng ZK, Shen XM, et al. Diagnostic value of fluorine-18 fluorodeoxyglucose positron emission tomography/ computed tomography in patients with fever of unknown origin. Eur J Intern Med 22: 112-116, 2011.

23. Kubota K, Nakamoto Y, Tamaki N, et al. FDG-PET for the diagnosis of fever of unknown origin: a Japanese multi-center study. Ann Nucl Med 25: 355-364, 2011.

24. Stumpe KD, Urbielli M, Steinert HC, et al. Whole body positron emission tomography using fluorodeoxyglucose for staging of lymphoma: effectiveness and comparison with computed tomography. Eur J Nucl Med 25: 721-728, 1998.

25. Schaefer NG, Hany TF, Taverna C, et al. Non-Hodgkin lymphoma and Hodgkin disease: coregistered FDG PET and CT at staging and restaging--do we need contrast-enhanced CT? Radiology 232: 823-829, 2004.

26. Isasi CR, Lu P, Blaufox MD. A metaanalysis of 18F-2-deoxy-2fluoro-D-glucose positron emission tomography in the staging and restaging of patients with lymphoma. Cancer 104: 1066-1074, 2005.

27. Jerusalem G, Beguin Y, Najjar F, et al. Positron emission tomography (PET) with 18F-fluorodeoxyglucose (18F-FDG) for the staging of low-grade non-Hodgkin's lymphoma (NHL). Ann Oncol 12: 825-830, 2001.

28. Alam K, Jain A, Maheshwari V, et al. Fine-needle aspiration cytology diagnosis of non-Hodgkins lymphoma in a resourcechallenged environment. Diagn Cytopathol 39: 461-467, 2011.

29. Hiraoka N, Yokote T, Nakayama-lchiyama S, et al. High fever and shock induced by interferon- $\gamma$ and interleukin- 6 produced by adult
T-cell leukaemia/lymphoma cells. Leuk Res 34: e290-e291, 2010.

30. Li Z, Liu WP, Tang Y, et al. Splenic T-cell and NK-cell lymphomas: a clinicopathologic and immunophenotypic analysis of 9 cases. Zhonghua Xue Ye Xue Za Zhi 28: 217-222, 2007.

31. Takahashi N, Chubachi A, Kume M, et al. A clinical analysis of 52 adult patients with hemophagocytic syndrome: the prognostic significance of the underlying diseases. Int J Hematol 74: 209213, 2001

32. Takahashi N, Miura I, Chubachi A, et al. A clinicopathological study of 20 patients with T/natural killer(NK)-cell lymphomaassociated hemophagocytic syndrome with special reference to nasal and nasal-type NK/T-cell lymphoma. Int J Hematol 74: 303308, 2001.

33. Willemze R, Jansen PM, Cerroni L, et al. Subcutaneous panniculitis-like T-cell lymphoma: definition, classification, and prognostic factors: an EORTC Cutaneous Lymphoma Group study of 83 cases. Blood 111: 838-845, 2008.

34. Han AR, Lee HR, Park BB, et al. Lymphoma-associated hemophagocytic syndrome: clinical features and treatment outcome. Ann Hematol 86: 493-498, 2007.

35. Miyahara M, Sano M, Shibata K, et al. B-cell lymphomaassociated hemophagocytic syndrome: clinicopathological characteristics. Ann Hematol 79: 378-388, 2000.

36. Sano T, Sakai H, Takimoto K, et al. Rituximab alone was effective for the treatment of a diffuse large B-cell lymphomaassociated with hemophagocytic syndrome. Int J Clin Oncol 12: 59-62, 2007.

37. Shimada K, Kinoshita T, Naoe $T$, et al. Presentation and management of intravascular large B-cell lymphoma. Lancet Oncol 10: 895-902, 2009.

38. A predictive model for aggressive non-Hodgkin's lymphoma. The International Non-Hodgkin's Lymphoma Prognostic Factors Project. N Engl J Med 329: 987-994, 1993.

39. Chihara D, Oki Y, Ine S, et al. Analysis of prognostic factors in peripheral T-cell lymphoma: prognostic value of serum albumin and mediastinal lymphadenopathy. Leuk Lymphoma 50: 19992004, 2009.

40. Beltran B, Quinones P, Morales D, et al. Different prognostic factors for survival in acute and lymphomatous adult T-cell leukemia/ lymphoma. Leuk Res 35: 334-339, 2011.

41. Lindh J, Lenner P, Osterman B, et al. Prognostic significance of serum lactic dehydrogenase levels and fraction of S-phase cells in non-Hodgkin lymphomas. Eur J Haematol 50: 258-263, 1993.

42. Gordon LI, Andersen J, Colgan J, et al. Advanced diffuse nonHodgkin's lymphoma. Analysis of prognostic factors by the international index and by lactic dehydrogenase in an intergroup study. Cancer 75: 865-873, 1995.

43. Lin R, Wang SS, Zhou SY, et al. Analysis of alkaline phosphatase staining results in 238 cases of malignant lymphoma. Di Yi Yun Yi Da Xue Xue Bao 23: 1097-1098, 2003.

44. Chihara D, Oki Y, Onoda H, et al. High maximum standard uptake value (SUVmax) on PET scan is associated with shorter survival in patients with diffuse large B cell lymphoma. Int J Hematol 93: 502-508, 2011.

45. Chan WKS, Au WY, Wong CYO, et al. Metabolic activity measured by F-18 FDG PET in natural killer-cell lymphoma compared to aggressive B- and T-cell lymphomas. Clin Nucl Med 35: 571$575,2010$.

46. Ngeow JYY, Quek RHH, Ng DCE, et al. High SUV uptake on FDG-PET/CT predicts for an aggressive B-cell lymphoma in a prospective study of primary FDG-PET/CT staging in lymphoma. Annals of Oncology 20: 1543-1547, 2009.

(C) 2012 The Japanese Society of Internal Medicine http://www.naika.or.jp/imonline/index.html 